\title{
Algebre senza base finita (I) $\left(^{*}\right)$.
}

\author{
Memoria di Iacopo Barsottr (a Pisa).
}

Sunto. - Si tratta assiomaticamente dei sistemi lineari sopra un corpo qualsiasi, con parti. colare riguardo alla separazione delle proprietà derivanti dal postulato della scelta.

\section{INTRODUZIONE}

La teoria delle algebre si è interessata quasi esclusivamente delle algebre associative (per quanto riguarda il prodotto), lineari, distributive, ed a base finita. Scopo del presente lavoro è di stabilire le principali proprietà delle algebre che si ottengono lasciando cadere l'ultima ipotesi, e di mostrare l'importanza che assumono in tale caso alcuni concetti che nelle algebre a base finita sono di importanza minima.

In successivi lavori si darà maggiore sviluppo alle parti che offrono più interesse, e lá presente nota ne costituisce la premessa necessaria; questa viene pubblicata in tre parti:

I. - Sistemi lineari:

II. - Algebre senzi base finita;

III. - Algebre regolari e algebre booleane.

L'un co lavoro sullargomento ('), a mia conoscenza, è il n. [4] (ofr. biblio. grafia), dove il WEDDERBURN, piuttosto che cercare le proprietà che valgono per le algebre senza base finita, si propone di sostituire l'ipotesi dell'esistenza della base finita con altre da cui si possano dedurre le stesse proprietà principali che valgono per le algebre a base finita $\left({ }^{2}\right)$.

\section{GAP. I - Sistemi lineari.}

1.1. Definizione; indipendenza dei postulati. - Si dirà sistema lineare, o brevemente sistema, nel corpo numerico $\Gamma$, un insieme $A$ di enti, che soddisfa alle seguenti proprietà :

(1) Se (a, b) è una coppia non ordinata di elementi di $\mathrm{A}$, è definito l'elemento $\mathrm{S}(\mathrm{a}, \mathrm{b}) d i \mathrm{~A}, e$ se e è elemento di $\mathrm{A}, \grave{e}$ :

$$
S(S(a, b), c)=S(a, S(b, c)) .
$$

(*) Estratto dalla tesi di laurea (Pisa, Scuola Normale Superiore, 1942).

(1) Altri lavori, una cui lista sara data nella parte III, trattano l'argomento facendo uso della teoria della rappresentazione. Tale teoria non viene usata nella presente nota (eccetto che per la definizione delle algebre regolari), e tutti i risultati vengono ottennti col * metodo diretto".

(2) Si è usato qualohe volla il postulato di ZERMreso; i risultati raggiunti mediante esso sono stati tenuti separati dal resto fella trattazione, e $j$ numeri ohe vi si riferiscono sono contrassegnati con una $Z$. 
(2) $S e \alpha, \beta$ sono numeri di $\Gamma$, e a, b elementi di A. e $(x$, a) è una coppia non ordinata, esiste l'elemento $\mathrm{D}(\alpha$, a) per cui ̀̀ :

$$
\begin{gathered}
D(\alpha, D(\beta, a))=D(\alpha \beta, a) ; \quad D(\alpha, S(a, b))=S(D(\alpha, a), D(\alpha, b)): \\
\\
D(\alpha+\beta, a)=S(D(\alpha, a), D(\beta, \alpha)) .
\end{gathered}
$$

(3) Se a, b sono elementi di A, esiste in $\mathrm{A}$ un elemento $\mathrm{x}$ tale che:

(4) Si ha:

$$
S(a, x)=b \text {. }
$$

per ogni a $d i \mathrm{~A}$.

$$
D(1, a)=a
$$

Per aniformarsi all' uso corrente si porrì:

$$
S(a, b)=a+b ; \quad D(\alpha, a)=\alpha a .
$$

Le proprietà scritte divengono allora:

$$
\begin{gathered}
a+b=b+a ; \quad(a+b)+c=a+(b+c) . \\
\alpha a=\alpha \alpha ; \quad \alpha(\beta a)=(\alpha \beta) a ; \quad \alpha(a+b)=\alpha a+\alpha b ; \\
(\alpha+\beta) \alpha=\alpha a+\beta a .
\end{gathered}
$$

(7) Se a, b sono elementi di $\mathrm{A}$, esiste un elemento $\mathrm{x}$ di A tale che:

$$
a+x=b \text {. }
$$

$$
1 \alpha=a \text {. }
$$

Le proprieta (7), (8) sono sostituite, nel caso dei sistemi a base finita, dalla proprieta :

(9) Esiste un numero finito di elementi $\mathrm{a}_{1}, \mathrm{a}_{2}, \ldots, \mathrm{a}_{\mathrm{n}}$ tali che ogni elemento $\mathrm{k}$ di A si può mettere, ed in un sol modo, solto la forma:

$$
k=\alpha_{1} a_{1}-1 \alpha_{2} \alpha_{2}+\ldots+\alpha_{n} \alpha_{n}, \text { con } \alpha_{1}, \ldots, \alpha_{n} \text { nmmeri: }
$$

Se allora

$$
\begin{gathered}
k=\alpha_{1} a_{1}+\ldots+\alpha_{n} a_{n}, \quad h=\beta_{4} a_{1}-\ldots+\beta_{n} a_{n}, \\
q=\left(\alpha_{1}-\beta_{1}\right) a_{1}+\ldots+\left(\alpha_{n}-\beta_{n}\right) a_{n},
\end{gathered}
$$

si ha:

$$
h+q=k,
$$

e la (7) ̀̀ soddisfatta; la (8) discende immediatamente.

Poichè la compatibilità e l'indipendenza dei (5), (6). e dei (5)), (6), (9) è già nota, rimane da dimostrare:

a) che i (5), (6), (7), (8) sono compatibili;

b) che la negazione di (7) è compatibile con i (5), (6), (8):

c) che la negazione di (8) è compatibile con i (5), (6), (7).

La dimostrazione di a) è immediata, se si considera che i $(7)$ e $(8)$ sono conseguenza di (9), e ehe i (5), (6), (9) sono compatibili. 
Dimostrazione di $b$ ): sia $A$ l'insieme formato dagli elementi $a_{1}, a_{2}, a_{3}$, con le operazioni definite nel modo seguente:

$$
\begin{gathered}
a_{i}+a_{j}=a_{i}+a_{i}=a_{3} \text { se } i \neq j ; \quad a_{i}+a_{i}=a_{i}: \\
\alpha a_{i}=a_{i} \alpha=a_{i}(\alpha \text { numero di un dato corpo }) .
\end{gathered}
$$

Tale insieme soddisfa ai (5). come si constata con la prova diretta; inoltre esso soddisfa anche ai (6), (8), ma non al (7), poichè non puo essere

$$
a_{1}+a_{i}=a_{2} \text {. }
$$

Dimostrazione di $c$ ): $\operatorname{sia} A$ l'insieme formato dagli elementi $a, b$, e pongasi :

$$
a+b=b+a=a ; \quad b+b=a+a=b ; \quad \alpha a=a \alpha=\alpha b=b \alpha=b
$$

( $\alpha$ numero del corpo $C(2)$ ).

$A$ soddisfa evidentemeate al primo dei (5), ed anche al secondo, come si constata con la prova diretta; esso soddisfa inoltre anche ai (6), (7), ma non al $(8)$, poichè

$$
1 a=b .
$$

In tal modo resta dimostrata la compatibilita ed indipendenza dei postu. lati (5). (6), (7), (8).

Si potrebbe avere il dubbio ohe $i$ (7), (8) fossero equivalenti al (9), di fronte ai (5), (6): ciò in realtà non accade, come si vede dal teorema (14).

\subsection{Elemento zero e operazioni sui sistemi.}

(10) Teorema. - Se A è un sistema, esiste un solo elemento e tale che per ogni a di A sia.

$$
a+e=a .
$$

Dim. - Sia $e$ tale che $a+e=a(7)$ con $a$ determinato; sia $b$ un elemento qualsiasi di $A$, e $c$ sia tale che $a+c=b$; allora:

$$
b+e=a+c+e=a+c=b,
$$

onde l'elemento $e$ gode della proprietà descritta. Che sia unico deriva dal fatto che se $f$ ha lat stessa proprietà di $e$, è anche:

$$
e+f=e, \quad e+f=f, \text { onde } e=f, \quad \text { c. } \mathbf{v} \text {. d. }
$$

(11) Teorema. - Se $\mathbf{a}+\mathbf{x}=\mathbf{a}, \grave{e} \mathbf{x}=\mathrm{e}$.

Dim. - Se infatti $b$ eे un elemento qualsiasi di $A$, esiste certo (7) un elemento $c$ tale che $a+c=b$, ed allora:

$$
b+x=a+c+x=a+c=b \text {, onde }(10) x=e, \quad \text { c. v. d. }
$$

(12) Teorema. - Se a è elemento di A, è:

$$
0 a=e .
$$

Infatti (6), (8): $0 a+a=(0+1) a=a$, onde (11) $0 a=e$. 
L.' elemento $e$ dicesi lo zero del sistema, e și può indicare con 0 senza generare confusione. L'elemento $x$ tale che $a+x=0$ si indicherd con $-a$.

Si porrà $a-b=a+(-b)$; si vede immediatamente che se $a+b=c$, è $b=c-n$. È anche :

$$
(-1) a=-a \text {. }
$$

(14) Teorema. - Esistono sistemi lineari tali che per ogni intero $\mathrm{n}>0$ si possono trovare $\mathrm{n}$ elementi $\mathrm{a}_{4}, \ldots, \mathrm{a}_{\mathrm{n}}$ fro loro linearmente indipendenti $\left({ }^{3}\right)$.

Dim. - Sia $A$ l'insieme delle successioni $; \alpha_{1}, \alpha_{2}, \ldots$ : ove gli $\alpha_{i}$ sono numeri del corpo $\Gamma$, essendovi però, in ogni successione, solo un numero finito di essi che siano $\neq 0$. Se

pongasi :

$$
\left.a=i \alpha_{1}, \alpha_{2}, \ldots\right\}, \quad b=i \beta_{1}, \beta_{2}, \ldots,
$$

$$
a+b=\left\{\alpha_{1}+\beta_{1}, \alpha_{2}+\beta_{2}, \ldots\right\}, \quad \alpha a=\left\{\alpha \alpha_{1}, \alpha \alpha_{2}, \ldots\right\}=a \alpha .
$$

L'insieme $A$ diviene in tal modo un sistema; pongasi :

ove

$$
a_{i}=i \alpha_{1}^{(i)}, \alpha_{2}^{(i)}, \ldots i
$$

sè è :

$$
\alpha_{r}^{(i)}=\left\{\begin{array}{lll}
0 & \text { se } & i \neq r \\
1 & \text { se } & i=r
\end{array}\right.
$$

$$
\mu_{1} a_{1}+\ldots+\mu_{n} a_{n}=0, \text { con } \mu_{1}, \ldots, \mu_{n}
$$

numeri, dovrà essere:

$$
\left.\mid \mu_{1}, \mu_{2}, \ldots, \mu_{n}, 0,0, \ldots\right\}=\{0,0, \ldots\} \text {, onde } \mu_{i}=0, \quad \text { e. } v . d \text {. }
$$

Se $B$ e un sub-insieme di $A$, chiuso rispetto alle operazioni $S$ e $D$, si dirà che $B$ è un sub-sistema di $A$. Esso è certo un sistema (13). È palese il seguente

(15) Teorema. - L'insieme degli elementi comuni a due sub-sistemi $\mathrm{H}, \mathrm{K}$ di A $\grave{e}$ un sub-sistema di $\mathrm{A}$, che sarà indicato con $\mathrm{H} \circ \mathrm{K}$, e si dirà intersezione $d i \mathrm{H} e \mathrm{~K}$.

Si dirǹ somma di due sub-sistemi $H, K$ di $A$ l'insieme di tutti gli elementi di $A$, ciascuno dei quali si può esprimere come somma di un elemento di $H$ e uno di $K$; detto $L$ tale insieme, si porrà :

$$
L=H+K
$$

si constata immediatamente che $L$ \& un sub-sistema di $A$.

Si dirà sub-sistema nullo di $A$ e si indicherà con 0 il sistema formato dall'elementu zero: due sub-sistemi si diranno disgiunti se la loro intersezione a) il sub-sistema nullo di $A$; si diranno complementari in $A$ se sono disgiunti, e se la loro somma ̀̀ $A$.

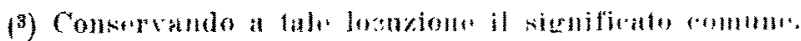


Dei sub-sistemi $A_{1}, \ldots, A_{r}$ di $A$ si diranno complementari in $A$ se ciascuno è complementare in $A$ della somma degli altri.

Siano ora $A, B$ due sistemi senza elementi comuni, $\theta$ si consideri l'insieme delle coppie $(a, b)$, con $a$ elemento di $A$ e $b$ di $B$; pongasi :

$$
(a, b)+\left(a^{\prime}, b^{\prime}\right)=\left(a+a^{\prime}, b+b^{\prime}\right), \quad \alpha(a, b)=(\alpha a, \alpha b),
$$

con evidente significato dei simboli. Tale insieme, con le operazioni cosi definite, è un sistema, che si indicherà con $A+B$.

Premesso che due sistemi si dicono equivalenti se si puo porre fra i loro elementi una corrispondenza biunivoca tale che il corrispondente di $\alpha a$ sia $\alpha a^{\prime}$, e quello di $a+b$ sia $a^{\prime}+b^{\prime}$, qualora $a^{\prime \prime}, b^{\prime}$ siano i corrispondenti di $a, b$ rispettivamente, e $\propto$ sia numero, si ha il

(16) Teorema. - Se A, B sono due sistemi, A + $\mathrm{B}$ contiene due sub-sistemi complementari in $\mathrm{A}+\mathrm{B}$, equivalenti rispettivamente ad $\mathrm{A}, \mathrm{B}$.

Essi sono infatti $\mathrm{i}$ sub-sistemi formati dagli elementi del tipo $(a, 0)$ e $(0, b)$ rispettivamente.

(17) Teorema. - Condizione necessaria e sufficiente affinchè un elemento della somma di due sub-sistemi $\mathrm{H}, \mathrm{K}$ di A sia rappresentabile in un sol modo come somma di un elemento di $\mathrm{H}$ e uno di $\mathrm{K}$, è che sia $\mathrm{H} \circ \mathrm{K}=0$.

Dim. - Sia $H \circ K=0$, e si supponga ohe

$$
a=h+k=h^{\prime}+k^{\prime},
$$

con evidente significato dei simboli; allora

$$
\left(h-h^{\prime}\right)+\left(k-k^{\prime}\right)=0, \quad \text { ossia } \quad h-h^{\prime}=k^{\prime}-k,
$$

e perciò, essendo $h-h^{\prime}$ elemento ải $H$, e $k^{\prime}-k$ di $K$, è $h=h^{\prime}, k=k^{\prime}$, e la condizione è sufficiente. È poi necessaria, perchè se $H \circ K=D \neq 0$, e $d \neq 0$ è elemento di $D$, si può porre

$$
h+k=(h+d)+(k-d)
$$

c. v. d.

Se $B$ è un sub-sistema proprio di $A$ (ossia $B<A$ ), si può, come è noto, ripartire gli elementi di $A$ in classi $\bmod B$, e definire per tali classi la somma e il prodotto per un numero, ottenendo un sistema differenza:

$$
A-B \text {. }
$$

1.3. Sistemi sommatori. - Nel presente numero, tutti i sistemi che si considerano sono sub-sistemi di uno stesso sistema.

Un sistema $A$ si dirà sommatorio se, essendo $B$ un suo qualsiasi subsistema, esisto in $A$ un complementare di $B$.

(18) Teorma. - La somma di due sistemi sommatori disgimnti è un sistema sommatorio.

Dim. - Siano $A$ e $B$ i due sistemi sommatori disgiunti, e $O=A+B$. Sia $D$ un sul-sistema di $C ; \therefore D \geq D \circ A+D \circ B$; 
se $D=D \circ A+D \circ B$, detti $A^{\prime}$ e $B^{\prime}$ dei complementari di $D \circ A$ e $D \circ B$ in $A, B$ rispettivamente, e posto $D^{\prime}=A^{\prime}+B^{\prime}$, è chiaro che $D, D^{\prime}$ sono com. plementari in $C$.

Se invece $D>D \circ A+D \circ B$, un elemento $d^{*}$ di $D$ e non di $D \circ A+D \circ B$ non può essere della forma $p^{*}+q$, con $p^{*}$ elemento di $A$ e non di $D \circ A$, e $q$ elemento di $D \circ B$, poichè $p^{*}$ sarebbe elemento di $D$, e quindi di $D \circ A$; analogamente non può essere della forma $p+q^{*}$, onde sarà necessariamente $d^{*}=p^{*}+q^{*}$, con $p^{*}, q^{*}$ elementi di $A, B$ rispettivamente, ma non di $D$.

Se allora $A^{\prime}, B^{\prime}$ sono i sistemi sopra definiti, esistono (17) un solo $p$ di $D \circ A$ ed un solo $p^{\prime}$ di $A^{\prime}$ tali che $p^{*}=p+p^{\prime}$, e analogamente scambiando i $p$ con i $q$ e gli $A$ con i $B$; perciò, per ogni elemento $d^{*}$ di $D$ e non di $D \circ A+D \circ B$, esiste un elemento $k=p+q$ di $D \circ A+D \circ B$, e due ele. menti $p^{\prime}, q^{\prime}$ di $A^{\prime}, B^{\prime}$ perfettamente determinati, tali che $d^{*}=p^{\prime}+q^{\prime}+k$; inoltre, l'insieme degli elementi $p^{\prime}$ di $A^{\prime}$ per ognuno dei quali esiste qualche $q^{\prime}$ di $B^{\prime}$ tale che $p^{\prime}+q^{\prime}$ sia elemento di $D$, è ù sistema, e ad ogni suo elemento corrisponde un solo $q^{\prime}$, perohè sè anche $p^{\prime}+\bar{q}^{\prime}$ è elemento di $D$, $q^{\prime}-\bar{q}^{\prime}=\left(p^{\prime}+q^{\prime}\right)-\left(p^{\prime}+\bar{q}^{\prime}\right)$ è elemento di $D \circ B$, e quindi è $=0$; sia $A^{\prime \prime}$ tale sistema, e $B^{\prime \prime} l^{\prime}$ analogo in $B^{\prime}$.

Sia $B_{1}$ complementare di $K=D \circ B+B^{\prime \prime}$ in $B$, e sia $H=A^{\prime}+B_{1}$. Per quanto detto sopra, $H$ è disgiunto da $D$; inoltre, se $c$ è elemento dí $C$, si può porre $c=a+b$, con $a$ in $A$ e $b$ in $B$; poi, $a=\bar{a}+a^{\prime}$, con $\bar{a}$ in $D \circ A$ e $a^{\prime}$ in $A^{\prime}$, e $b=k+b_{1}$, con $k$ in $K$ e $b_{1}$ in $B_{1}$; infine, $k=\bar{b}+b^{\prime \prime}$, con $\bar{b}$ in $D \circ B$ e $b^{\prime \prime}$ in $B^{\prime \prime}$; sia allora $a^{\prime \prime}$ l'elemento che corrisponde (nella corrispondenza sopra stabilita) a $b^{\prime \prime}$ in $A^{\prime \prime} \leq A^{\prime}$; si avrà :

$$
\begin{gathered}
c=a+b=\bar{a}+a^{\prime}+k+b_{1}=\bar{a}+a^{\prime}+\bar{b}+b^{\prime \prime}+b_{1}+a^{\prime \prime}-a^{\prime \prime}= \\
=\left[\bar{a}+\bar{b}+\left(a^{\prime \prime}+b^{\prime \prime}\right)\right]+\left[a^{\prime}+b_{1}-a^{\prime \prime}\right],
\end{gathered}
$$

e la prima di queste parentesi è elemento di $D$, mentre la seconda è ele. mento di $H$, onde $D, H$ sono complementari in $C$, c. v. d.

(19) Teorema. - Un sub-sistema di un sistema sommatorio è sommatorio.

Dim. - Sia $B<A$, con $A$ sommatorio, e sia $C$ un complementare di $B$ in $A$.

Sia $D$ un suh-sistema di $B$, e sia $E$ n complementare di $D+C$ in $A$. Ogni elemento $e$ di $E$ si pui porre, in un sol modo, nella forma $e=b+c$, con $b$ in $B \in c$ in $C$.

L'elemento $b$, se $\dot{\theta} \neq 0$, non appartiene certo a $D$; sia $H$ l'insieme degli elementi $b$ di $B$ per ognuno dei quali esiste un $c$ di $C$ tale che $b+c$ sia elemento di $E$.

$H$ è un sistema disgiunto da $D$; sia $b$ un elemento di $B:$ si può porre $b=e+d+c$ con $e$ in $E, d$ in $D, c$ in $C$, con $e, d, c$ perfettamente determinati; si può poi porre: $e=h+c^{\prime}$, con $h$ in $H, c^{\prime}$ in $C$, e $h, c^{\prime}$ perfettamente determinati; ne discende :

$$
b=h+c^{\prime}+d+c=h+d+\left(c^{\prime}+c\right), \quad \text { e } \quad c^{\prime}+c=0
$$

essendo $h$ e $d$ in $B$, donde $b=h+d$, e perciò $H+D=B$, c. v. $d$. 
(20) Corollario. - L'intersezione di due sistemi, di cui uno sia sommatorio. è un sistema sommatorio.

(21) Teorema. - La somma di due sistemi sommatori e un sistema sommatorio.

Dim. - Sia $A=B+C$, con $B, C$ sommatori, e $D=B \circ C$. Sia $E$ com. plementare di $D$ in $B ; E \in C^{\prime}$ sono disgiunti, e la loro somma è $A$; poichè (19), (20) $E$ e $D$ sono sommatori, tale è $A$ (18), e. v. d.

(22) Tеовема. - La somma di due sistemi, uno dei quali sia non sommatorio, è non sommatoria.

Deriva da (19) per assurdo.

È noto che ogni sistema a base finita è sommatorio.

Si ha infine, con dimostrazione immediata, il

(23) Teorema. - Se A, B sono sistemi disgiunti, e $\mathrm{A}+\mathrm{B}=\mathrm{C}, \mathrm{C}-\mathrm{B}$ è equi. valente ad A.

1.4. Base di un sistema. - Si dira base di un sistema $A$, un insieme $T$ di elementi di $A$, tali che nessuno di essi sia combinazione (lineare omogenea) di altri, e tali che ogni elemento di $A$ sia combinazione di elementi di $T$ (si intende, in numero finito).

Si constata immediatamente che

(24) La somma di due sub-sistemi disginnti di un sistema A, che sicno dotati di base, è dotaia di base.

E naturale che, uscendo dal campo dei sistemi a base finita, oi si trovi ad affrontare quelle difficolta concettuali che si incontrano nello studio degli insiemi infiniti. Così, si dorranno distinguere basi finite, infinite, ordinate, ben ordinate; e di una hase infinita si potrà considerare la potenza, che potrì, o no, essere un alef, ed eventualmente il tipo d'ordine, che potrà, o no, essere un numero ordinale.

(25) Limma. - L'insieme dei sub-insiemi finiti di un insieme che ha per potenza un alef, ha per potenza quell'alef.

Dim, - Sia $I$ l'insieme dato, e $K$ l'insieme dei sub-insiemi finiti di $I$. Se $K_{n}$ è l'insieme dei sub-insiemi di $I$ che constano di $n$ elementi, sarà

$$
K=\sum_{i}^{\infty} K_{n}
$$

La potenza di $K_{n}$ è $\mathbf{\$}_{\omega}^{n}=\mathbf{\$} \omega$, qualora $\mathbf{\aleph}_{\omega}$ sia la potenza di $I$. Per ogni intero positivo $n$ è perfettamente determinata una corrispondenza biunivoca fra $K_{\imath}$ ed $I$, onde si può mettere in corrispondenza biunivoca $K$ con l'in. sieme delle coppie $(n, J)$, con $n$ intero positivo e $J$ elemento di $I$; ma l'insieme di tali coppie ha la potenza

$$
\mathbf{N}_{0} \mathbf{\$}_{\omega}=\mathbf{N}_{\ldots,} \text {, onde } \mathbf{\aleph}_{\omega} \text { è la potenza di } K, \quad \text { c. } \mathbf{v} \text { d. }
$$

(26) Teorema. - Se $\mathbf{\aleph}_{i}, \mathbf{\aleph}_{j}$ sono le potenze di due basi di un sistema, è $\mathbf{\aleph}_{i}=\mathbf{N}_{j}$. 
Dim. - Siano $T_{i}, T_{j}$ due basi di $A$, e $T_{i}$ abbia la potenza $\aleph_{i}$, e $T_{j}$ la potenza $\mathbb{\aleph}_{j}$. Se $t$ è elemento di $T_{i}$, esso sarà combinazione di elementi di $T_{j}$, con coefficienti tutti $\neq 0$; sia $D_{t}$ l'insieme di tali elementi.

Si possono dividere gli elementi di $T_{i}$ in classi, ponendo $t, s$ nella stessa classe $o$ in classi diverse, secondochè $D_{t}=D_{s}$ o $D_{t} \neq D_{s}$; tali classi sono a due a due disgiunte; se $S$ è l'insiene di tali classi, e $S^{\prime}$ l'insieme dei $D_{t}$. al variare di $t$ in $T_{t}$, si può porre una corrispondenza biuniqoca fra $S$ e $S^{\prime}$; la potenza di $S^{\prime}$ è $(25) \leq \mathbf{\aleph}_{j}$. Si consideri l'insieme $R$ delle coppie $(\$, n)$, con $n$ intero positivo e $\mathcal{S}$ elemento di $S$ : essendo $S^{\prime}$, e quindi $S$, ben ordinabile, la sua potenza è un $\aleph_{k}$, e quella di $R$ è $\aleph_{0} \aleph_{k}=\aleph_{k}$, e poichè $T_{i}$ si può mettere in corrispondenza biunivoca con un sub-insieme di $R$, sarà :

$$
\mathbf{N}_{i} \leq \mathbf{N}_{k} \leq \mathbf{N}_{j}
$$

In maniera analoga si dimostra che

$$
\mathbf{N}_{i} \geq \mathbf{N}_{j} \text {, onde } \mathbf{\aleph}_{i}=\mathbf{\aleph}_{j}\left({ }^{4}\right), \quad \text { c. } \mathbf{v} \text { d. }
$$

(27Z) Teonema. - Tutte le basi di un sistema hanno la stessa potenza.

Infatti il teorema di Zermelo assicura che esse sono ben ordinabili. (28Z) Teorema. - Ogni sistema è dotato di base ben ordinabile.

Dim. - Sia $A$ il sistema, e suppongasi di aver ben ordinato i suoi elementi.

Sia $T$ l'insieme degli elementi ciascuno dei quali non è combinazione di elementi che lo precedono. Evidentemente nessun elemento di $T$ è combinazione di altri elementi di $T$ che lo precedono, e ogni elemento di $A$ ma non di $T$ è combinazione di elementi di $T$ che lo precedono, poichè se esístessero elementi di $A$ ma non di $T$ per eui ciò non accadesse, detto $C$ il loro insieme, $C$ avrebbe un primo elemento $c$ che, non appartenendo a $T$, dovrebbe essere combinazione di elementi che lo precedono; ma ognuno di tali elementi, non appartenendo a $C$, sarebbe combinazione di elementi di $T$ ad esso non successivi, e quindi anche $c$ godrebbe di tale proprietà, contro l'ipotesi, c. r. d.

(29) Teorema. - Ogni sistema $i$ cui elementi formano un insieme ben ordinabile, è dotato di base.

(30) Teorema. - Se il sistema sommatorio A possiede una base T ben ordinabile, ogni sub-sistema di A possiede una base ben ordinabile.

Dim. - Sia $B$ un sub-sistema di $A$, e $C$ un suo complementare in $A$. Ogni elemento $t$ di $T$ si puó porre sotto la forma $t=b+c$, eon $b$ in $B$ e $c$ in $C$, in un sol modo (17); sia $S$ l'insieme di tutti i detti $b$, e si ordini $S$ come segue: si faccia corrispondere ad $s$ di $S$ il primo fra gli elementi $t$ di $T$ che si possono mettere sotto la forma $t=s+c$, con $c$ in $C$; la corrispondenza è evidentemente biunivoca, e perciò, se si impone che essa sia simile, $S$ rimane ben ordinato; si estragga da $S$, col procedimento nsato nella

(4) Si rieordi che per gli alef vale la trieotomia. 
dimostrazione di (28Z), un sub-insieme $S^{\prime}$ di elementi ognuno dei quali non sia combinazione di altri, e tali che ogni elemento di $S$ sia combinazione di elementi di $S^{\prime}$ che lo precedono.

Se allora $b$ è elemento di $B$, esso sarà combinazione di elementi di $T$, e sarà perciò della forma $s+c$, con $c$ in $C$ e quindi nullo, e $s$ combinazione di elementi di $S$, e perciò di $S^{\prime}$, e. v. d.

(31) Teorema. - Ogni sistema dotato di base ben ordinabile è sommatorio. Dim. - Sia $T$ la base ben ordinata di $A$, e sia $B$ un sub-sistema di $A$.

Per ogni elemento $t$ di $T$, sia $C_{t}$ il sistema che ha per base l'insieme degli elementi di $T$ che precedono $t$. Sia $C$ l'insieme degli elementi $t$ di $T$ ciascuno dei quali non appartiene a $C_{t}+B$, sia $D$ il sistema che ha per base $C$.

E $D \circ b=0$, poichè se l'elemento $b \neq 0$ di $B$ fosse elemento di $D$, sarebbe una combinazione di elementi di $C$ e di conseguenza l'ultimo fra gli elementi di $C$ di cui $b$ è combinazione (sia esso $h$ ) sarebbe elemento di $C_{h}+B$, contro l'ipotesi.

Si osservi poi che se $t$ è un elemento di $T$, ma non di $C$, esso è somma di un elemento di $B$ e di una combinazione di elementi di $C$ che precedono $t$, poichè se $H$ fosse l'insieme degli elementi di $T$ ma non di $C$ che non godessero di tale proprietà, il primo elemento di $H$ sarebbe somma di un elemento di $B$, e di una combinazione di elementi di $T$ (e quindi di $C$ ) ad esso precedenti, contro l'ipotesi.

Perciò, se $a$ è elemento di $A$, esso è combinazione di elementi di $T$, ed ì perciò somma di un elemento di $B$, e di una combinazione di elementi di $C$, ossia di un elemento di $B$ e di un elemento di $D$, onde $A=B+D$, e $D$ è complementare di $B$ in $A$. c. v. $d$.

Da (30), (31) si ha il

(32) Corollarto. - Ogni sub-sistema di un sistema dotato di base ben ordinabile, è dotato di base ben ordinabile.

$\mathrm{E}$ da $(287),(31)$ :

(33Z) Trorema. - Ogni sistema è sommatorio.

1.5. Osservazioni. - Le nozioni di sistema sommatorio, e di sistema do. tato di base, non hanno, nel caso di sistemi a base finita, alcuna importanza, in quanto tali sistemi sono sempre sommatori e dotati di base. Esse sono invece, come si vedrà meglio nel seguito, fondamentali nel caso di sistemi non a base finita.

Dal punto di vista zermeliano esse perdono ogni importanza, in quanto, come si è visto, non caratterizzano speciali sistemi.

Dal punto di vista non zermeliano, al contrario, rimane adito, almeno nel modo in cui sono state svolte le dimostrazioni, al dubbio che esistano sistemi non a base ben ordinata, o non sommators.

Annali di Matematira, serie IV, Tomo XXVI. 
1.6. Esempio. - Sia $\omega_{i}$ in numero ordinale iniziale, vale a dire un numere ordinale tale che ogni insieme che abbia per tipo d'ordine un numero ordinale minore di $\omega_{i}$, abbia potenza minore della potenza di $\omega_{i}$.

Sia $a=\left(\alpha_{\omega}\right)$ un insieme di numeri del corpo numerico $\Gamma$, con $\omega$ variante nel campo dei numeri ordinali da 1 a $\omega_{i}$ compreso, e tale che vi sia solo un numero finito di $\alpha_{\omega}$ diversi da 0 .

Se $a=\left(\alpha_{\omega}\right), b=\left(\beta_{\omega}\right)$ sono due di tali elementi, e $\mu$ è un numero, si ponga:

$$
\mu a=a \mu=\left(\mu \alpha_{\omega}\right) ; \quad a+b=\left(\alpha_{\omega}+\beta_{\omega}\right) ;
$$

con queste definizioni, l'insieme di tali elementi è un sistema che ha per base (ben ordinabile) l'insieme degli elementi ciascuno dei quali ha nulli tutti gli $\alpha_{\omega}$, eccetto che uno, che è uguale ad 1 .

Il sistema cosı̀ definito è (31) sommatorio; esso sarà indicato con $S_{\omega_{i}}$, e la base sopraindicata si chiamerà fondamentale.

(34) Teonema. - Ogni sistema dotato di base ben ordinabile è equivalente ad un $\mathrm{S}_{w_{i}}$.

Dim. - La base avrà una certa potenza $\mathbf{\aleph}_{i}$, e si potrà perciò porre in corrispondenza biunivoca con l'insieme dei numeri ordinali minori di $\omega_{i}$, e quindi con la base fondamentale di $S_{\mathfrak{m}_{i}}$; di qui discende immediatamente il teorema.

(35) Teorema. - Se $\mathrm{S}_{\omega_{i}}$ è equivalente ad $\mathrm{S}_{\omega_{j}}$, ̀̀ $\omega_{\mathrm{i}}=\omega_{\mathrm{j}}$.

Dim. - Posta una corrispondenza di equivalenza fra $S_{\aleph_{i}}$ e $S_{\omega_{j}}$, alla base fondamentale di $S_{\omega_{i}}$ corrisponderà in $S_{\omega_{j}}$ una base di potenza $\widehat{S}_{i}$; ma tale base ha (26) la potenza $\aleph_{j}$, onde $\aleph_{i}=\$_{j}$, e perciò $\omega_{i}=\omega_{j}$, c. v. d.

Altre proprietà degli $S_{\left(\omega_{i}\right.}$ saranno stabilite nel n. 3.6. (36Z) TEorema. - Ogni sistema è equivalente ad un $\mathrm{S}_{\omega_{i}}$.

\section{BIBLIOGRAFIA (1)}

[1] SCORZA, Corpi numerici e algebre.

[2] ALberi, Modern higher Algebra (Philadelphia, 1938).

[3] A ispent, Structure of Algebras (Amer. Math. Soc. Coll. Public, 1939).

[4] Wedderburs, Alg. which do not possess a finite basis (Trans. Amer. Math. Soc., v. 26, p. 395,1924$)$.

[5] STone, Theory of representation for Boolean Alg. (ibia., v. 40, p. 37, 1936).

[6] WInNER, Certain formal invar. in Bnol. Alg. (ibid., v. 18, p. 65, 1917).

[7] Yon NewManN \& STONE, The determ, of repres. elem. in the residual classes of Bool. Alg. (Fund. Math., v. 25, p. 353, 1935). 